\title{
AUSENCIAS Y CONCENTRACIONES EN EL UNIVERSO MEDIÁTICO COSTARRICENSE
}

\section{ABSENCES AND CONCENTRATIONS IN THE COSTA RICAN MEDIA UNIVERSE}

\author{
Lidieth Garro Rojas* \\ Lilliana Solís Solís**
}

RESUMEN

\begin{abstract}
En este artículo se analizan las principales características del sistema de medios costarricense, entre estas, la concentración geográfica y la exclusión en el uso del espectro radioeléctrico. La información fue recabada y verificada mediante llamadas telefónicas, así como a través de Internet y redes sociales, entre los meses de octubre de 2018 y febrero de 2019. Entre otros aspectos se constata que la mayoría de los medios se ubican en la provincia de San José y están ausentes en 25 de los 82 cantones. También destaca que el 57\% de las televisoras transmiten a través de Internet o empresas de cable y no cuentan con acceso al espectro radioeléctrico.
\end{abstract}

PALABRAS CLAVE: DEMOCRACIA * MEDIOS DE COMUNICACIÓN DE MASAS * LIBERTAD DE EXPRESIÓN * CONCENTRACIÓN MEDIÁTICA

\section{ABSTRACT}

The main characteristics of the Costa Rican media system are analyzed, including geographic concentration and exclusion in the use of the radioelectric spectrum. The information was collected and verified through telephone calls, as well as the Internet and social networks, between October 2018 and February 2019. Among other aspects, it can be pointed out that most of the media is located in the Province of San José and is absent in 25 of the 82 cantons. Also, it stands out that $57 \%$ of the television broadcasters transmit over the Internet or cable companies and do not have access to the radioelectric spectrum.

KEYWORDS: DEMOCRACY * MASS MEDIA * RIGHT TO COMMUNICATE * MEDIA CONCENTRATION

* Escuela de Ciencias de la Comunicación Colectiva y Centro de Investigación en Comunicación, Universidad de Costa Rica, Costa Rica.

lidieth.garro@ucr.ac.cr

** Radio Universidad y Programa de Libertad de Expresión, Derecho a la Información y Opinión Pública, Universidad de Costa Rica, Costa Rica.

lilliana.solis@ucr.ac.cr 


\section{INTRODUCCIÓN}

En Costa Rica como en otros países democráticos, el ejercicio de la democracia está ligado a la posibilidad de contar con un sistema de medios de comunicación plural y diverso que sea capaz de garantizar la más amplia representación y participación a los habitantes.

Durante los últimos años en el país, se han realizado esfuerzos importantes para contar con una descripción amplia de los medios de comunicación con el fin de conocer su naturaleza, su ubicación y su alcance. Sin embargo, estos esfuerzos se han caracterizado por abordar aspectos parciales de los medios de comunicación, debido, en gran parte, a que se carecen de una base de datos unificada que indique cuántos son, dónde están y cuáles son las principales características de esos medios.

La carencia de esta información ha impedido un mayor escrutinio público en temas como propiedad, uso del espectro radioeléctrico, la distribución geográfica de las frecuencias del espectro radioeléctrico y otros asuntos que vinculan a los medios de comunicación con la democracia. Este artículo muestra parte de los resultados de una investigación en curso que busca construir indicadores de desarrollo mediático que permitan evaluar las condiciones existentes para el ejercicio de la ciudadanía comunicativa.

Dicha investigación dio inicio con el levantamiento de la información relativa a los medios de comunicación existentes en el país, a los que se ha denominado "universo mediático" costarricense y que está compuesto por todos los medios de comunicación identificados. Forman parte de ese universo, los medios de comunicación escritos, televisivos y radiofónicos, que emiten utilizando Internet, el espectro radioeléctrico o que circulan de forma impresa.

La inexistencia de una base de información completa y actualizada sobre dónde se encuentran y cuáles son las condiciones básicas de funcionamiento de los medios de comunicación es, en el entorno de esta sociedad altamente mediatizada, un síntoma de cómo, en tanto ciudadanos y ciudadanas, se posicionan frente a los medios de comunicación.
Es así que preguntas de gran actualidad como: ¿qué puede exigir la ciudadanía a los medios para que cumplan con su función social?, ¿qué debe conocer la ciudadanía sobre los medios de comunicación para poder ejercer su derecho a participar en ellos y en la sociedad democrática?, ¿qué responsabilidad deben tener los medios de comunicación en la construcción de sociedades democráticas?, quedan postergadas en la discusión ciudadana, en tanto se desconoce la información básica sobre los propios medios de comunicación.

Como sociedad se reconoce su presencia casi permanente, la importancia que adquieren al narrar los problemas, las tensiones, los conflictos y su devenir; algunos de ellos se han posicionado como referencia casi obligada al hablar de la actualidad y sus discursos se convierten en tema de disputa cultural o política, lo cual ha sido objeto de análisis académico.

Sin embargo, no se tiene una idea clara de cómo se configura y cambia ese entorno mediático. Los análisis que se realizan son generalmente relativos a medios de comunicación específicos, principalmente, aquellos considerados más tradicionales (en el sentido de permanencia en el tiempo), los cuales se producen en la región central de San José y con pretensiones de representar el acontecer del país o se concentran en coyunturas sociopolíticas o temporales bien delimitadas.

Pocas veces la conformación de la totalidad de los medios de comunicación ha sido tema para el análisis académico, aunque en los últimos años se ha puesto sobre la mesa, la disputa social por el uso del espectro radioeléctrico en la que han participado diversos actores sociales y políticos.

Estudios como los de Mastrini y Becerra (2009), Chinchilla (2013) y Fournier, Jiménez y Ochoa (2018) han procurado el análisis de los medios de comunicación desde las perspectivas de la concentración en conglomerados mediáticos; se destaca la falta de pluralidad y diversidad del sistema de medios de comunicación a partir de la concentración del espectro radioeléctrico en pocas manos. Robles y Voorend (2011) analizan además la vinculación de empresas de comunicación con capitales que 
se han relacionado con la reforma neoliberal en el país. Otras líneas temáticas de investigación han girado en torno al análisis de la legislación que regula a los medios de comunicación, con trabajos como los de Araya, Avendaño y Carazo (2013), y Boza (2014 y 2015).

Se trata de temas relevantes para el análisis de los medios de comunicación que atienden asuntos necesarios para la compresión de la conformación del universo mediático en las dimensiones económica y jurídica.

En 1990, Sandoval y Al-Ghassani elaboraban un inventario de medios de comunicación del país. El trabajo tuvo el mérito de ser el primer esfuerzo por conocer la cantidad y el tipo de medios que funcionaban en ese momento en Costa Rica. Entre otros temas, el informe reunía información en torno a tipo de propiedad, tarifas de publicidad, formato de impresión $y$ listas de accionistas de algunos medios de alcance nacional. Solo entre los medios impresos, el trabajo documentó la existencia de 328 medios de comunicación, entre los que se incluían publicaciones de avisos comerciales y revistas de instituciones de educación superior. El inventario fue una herramienta útil que ponía a disposición de las personas investigadoras y del país, un registro de la totalidad de los medios de comunicación costarricenses existentes en ese momento.

Pasaron más de 25 años sin que se contara con una actualización de la información. En 2014, Carazo retoma la idea de trabajar en el levantamiento de datos y recoge información específica acerca de los medios de comunicación comunitarios. La investigación desvela una realidad desconocida hasta entonces $y$ da cuenta de la existencia de al menos, 71 medios de comunicación que se reconocían como tales. El estudio recabó datos relativos a la ubicación y características generales de cada medio (la ubicación, el tipo de medio, el alcance geográfico, la audiencia estimada, el uso de redes sociales, la frecuencia, el tipo de uso del sitio web, los recursos utilizados en las redes sociales), los espacios periodísticos, las fuentes de financiamiento (el tipo de anunciantes, encargados de la producción publicitaria, los esfuerzos por contar con pauta), las instituciones vinculadas al medio (el tipo de institución vinculado a su creación, las funciones que cumple el medio, las motivaciones para contactar a la comunidad, la participación de la comunidad en el medio), la producción de contenidos propios (cantidad de programas, géneros, temáticas), entre otros asuntos. Se trató del primer esfuerzo sistemático por contar con información de los medios de comunicación autopercibidos como "medios comunitarios". La investigación de Carazo (2014) es el antecedente más cercano $y$ el listado de medios generado fue considerado en esta investigación.

\section{ABORDAJE}

Este artículo se ubica en la línea de investigación sobre ciudadanía comunicativa; un encuadre conceptual que parte del "reconocimiento de la capacidad de ser sujeto de derecho $y$ demanda en el terrero de la comunicación pública, y en el ejercicio de ese derecho" (Mata, 2006, p. 13), además de la ampliación de derechos que asiste a las personas y por tanto, coloca en la mirada del análisis, las condiciones existentes para el uso de los medios de comunicación en tanto plataforma para el ejercicio de esos derechos (Mata, 2006 y Córdoba, 2008).

La existencia de una diversidad de medios de comunicación que permita una polifonía de voces, aporta a la calidad de una democracia en la que los sujetos no son únicamente portadores de derechos (pasivos) sino que pueden acceder a los medios de comunicación para profundizar el ejercicio y demandar nuevos derechos.

Si bien, la evaluación de las condiciones para el ejercicio de la ciudadanía comunicativa abarca aspectos como las condiciones jurídicas para la acción, las políticas públicas y la actuación del Estado, la implicación ciudadana en la calidad de esos medios (Garro-Rojas, Monge y Solís, 2018) es requisito para contar con una idea amplia y completa de cómo se conforma ese universo de medios de comunicación al que se hace referencia.

Una de las condiciones que potencia el ejercicio de la ciudadanía comunicativa es la existencia de un sistema de medios caracterizado por la pluralidad. Según Lovatón (2014), 
el concepto de pluralidad implica una doble condición; por un lado, la pluralidad interna de los medios de comunicación que refiere al derecho que asiste a los periodistas a expresarse $y$ a difundir sus ideas e información en forma independiente, libre y plural dentro de su espacio laboral; $y$ por otro lado, la pluralidad externa que refiere a la necesidad de las sociedades democráticas de contar con medios de comunicación que expresen puntos de vista distintos y discrepantes, asimismo, que sean capaces de representar los puntos de vista de diversos actores sociales.

La pluralidad de los medios de comunicación se convierte en una de las condiciones para el ejercicio de un debate ciudadano amplio. La concentración de los medios en unas pocas empresas implicaría la falta de pluralidad externa, lo cual supone un daño potencial a la democracia y a las condiciones para el ejercicio de la ciudadanía comunicativa.

La concentración mediática es la condición opuesta a un sistema de medios plural. Fournier, Jiménez y Ochoa (2018) distinguen diversas formas de concentración de los medios de comunicación: 1) la concentración horizontal o monomedia, relacionada con la adquisición de diversos medios de comunicación por parte de una misma empresa; 2) la concentración vertical que consiste en la expansión en distintas fases de producción en un mismo medio de comunicación; 3) la concentración diagonal o conglomeral, que se presenta cuando un medio incursiona en otras áreas de la economía diferentes a la comunicación; 4) la concentración o integración multimedia, caracterizada por el control de distintos tipos de medios que funcionan sobre diversas plataformas; 5) la concentración internacional que describe la existencia de grupos de comunicación establecidos en diferentes países.

Este artículo se refiere a la concentración en su dimensión geográfica, no estudiada hasta ahora el entorno costarricense. La dimensión espacial en el estudio de los medios de comunicación puede mostrar que los lugares y los espacios no son neutrales y abren una nueva perspectiva de análisis que enriquece el estudio de los asuntos de actualidad referidos a la participación democrática, como por ejemplo, los movimientos sociales y los resultados electorales en elecciones recientes.

Sobre los medios de comunicación, no se recurre en este artículo a una definición taxativa de lo que son o deben ser, pues se reconoce que esta debe ser elaborada en una perspectiva histórica y acotada al contexto. En este sentido, es necesario reconocer que distintos fenómenos inciden en la conformación actual de los medios, muchos de ellos impulsados por el desarrollo tecnológico, la transnacionalización del capital, las nuevas formas de organización empresarial y los flujos de mercancías y personas.

La convergencia tecnológica y el desarrollo multimedial han favorecido nuevas posibilidades de enunciación por medio de la Internet $y$ las redes sociales, facilitando a las otrora audiencias pasivas convertirse en productoras de contenidos. Se ha consolidado la noción de multiplataforma como forma privilegiada de producción de contenidos para un usuario ávido de compartir, apropiarse y reelaborar los mensajes para sumarse al circuito de circulación.

Este complejo entorno obliga a hacer una definición de lo que en este artículo se considera como un "medio de comunicación". Por ello, interesa explicitar los criterios de selección utilizados para el levantamiento de la información presentada: los medios de comunicación pueden ser impresos, digitales, radiofónicos o televisivos. Usan para su emisión la plataforma impresa, digital, el espectro radioeléctrico o empresas de televisión por cable o varias de estas en forma simultánea. Son conocidos y autoreconocidos como tales en su contexto y funcionan con una periodicidad estable que es conocida por sus audiencias. Los contenidos de esas iniciativas deben ser renovadas con cierta regularidad y cuentan con un equipo de producción de al menos dos personas.

Una excepción a esta caracterización general son los medios que utilizan el espectro radioeléctrico. En este caso particular se consideraron todas las iniciativas.

En el caso de los medios digitales, estos deben utilizar una plataforma diferente a las 
redes sociales, por ejemplo, plataformas de emisión para radios online o canales de vídeo, tales como, Youtube o Vímeo. Además, producen contenidos propios y el último contenido, al momento de levantar la información, tenía menos de tres meses de haber sido actualizado.

El desarrollo de Internet ha favorecido que cada día aumente la cantidad de medios que utilizan esta plataforma para su emisión en forma simultánea: televisoras y radioemisoras, así como, medios escritos de larga trayectoria han aprovechado esa facilidad. En esos casos, se asume que la versión principal es la impresa o la que utiliza el espectro radioeléctrico y que el uso de medios digitales es complementario; por ejemplo, el Diario Extra de Costa Rica se catalogó como un medio de comunicación impreso y se reconoce la existencia del sitio web http:// www.diarioextra.com/

En el marco de este estudio, un medio de comunicación no es un programa radiofónico o televisivo específico, sin embargo, se da el caso de algunos programas noticiosos o de opinión que han logrado construir una trayectoria y una permanencia en redes sociales. En esos casos se toma como un medio de comunicación independiente la versión Web, que produce contenidos propios e interactúa con sus audiencias de forma independiente a la televisora o radio en la que también emite. Tal es el caso del programa "Nuestra Voz", que se emite por Radio Monumental y se incluye como el medio digital "ameliarueda.com" (https://www.ameliarueda.com).

La apertura de recursos Web ha abierto las posibilidades para el ejercicio de la libertad de expresión y ha permitido que cada vez más personas sean atraídas por la producción de contenidos. Sin embargo, no todos los productores de contenidos elaborados $y$ distribuidos a través de las redes sociales pueden ser considerados un medio de comunicación. Las publicaciones en redes o blogs de algunos productores (hoy reconocidos como "influencers") no constituyen, en el marco de esta investigación, un medio de comunicación. También se excluyen iniciativas conocidas como "periodismo ciudadano" que se presentan como perfiles en Facebook $u$ otras redes sociales $y$ que desarrollan dinámicas que les permite compartir información y opinión, generalmente sobre espacios geográficos reducidos. Asimismo, los medios o revistas institucionales, tales como, las producidas por las oficinas de relaciones públicas $y$, finalmente, las revistas de carácter académico que tienen como objetivo el diálogo disciplinar.

\section{METODOLOGÍA}

La recopilación de información se efectuó entre octubre de 2018 y febrero de $2019^{1}$. Para ello, se partió de varios listados preexistentes de medios de comunicación: el propuesto por Carazo (2017), del Colegio de Periodistas, del Programa de Libertad de Expresión y Derecho a la Información (PROLEDI) y de la Oficina de Divulgación de la Universidad de Costa Rica. También la información sistematizada por Rodríguez (2018) y Ochoa (2018) referente a concentración y asignación de publicidad estatal a medios. Estos listados parciales aportaron el insumo básico en el proceso de recopilación de información, la cual fue verificada y ampliada mediante la revisión de medios de comunicación en Facebook.

Un listado general de medios de comunicación fue verificado mediante la revisión de los datos de sus páginas Web, en la red social Facebook y por medio de llamadas telefónicas. Ese proceso de verificación permitió la elaboración de una base de datos con información relativa a:

$\diamond \quad$ Tipo de medio: televisivo, radiofónico, digital, prensa escrita, revista.

$\diamond \quad$ Plataforma: se refiere al canal principal de emisión.

$\diamond \quad$ Cobertura: se calificó según diversas opciones de respuesta (local: se distribuye en una sola ciudad o pueblo; regional: alcanza varias ciudades o diferentes cantones; nacional: llega a todo el país).

$\diamond \quad$ Cobertura en detalle: lugares principales (y específicos) en los que se distribuyen o alcanzan los medios.

$1 \quad$ El MSc. Allan Monge Cordero colaboró en la etapa de recolección y verificación de la información de los medios. 
$\diamond \quad$ Periodicidad: frecuencia de publicación del medio. La categoría solo aplica para medios impresos.

$\diamond \quad$ Tiraje: cantidad de ejemplares impresos de un medio. Al ser un dato delicado para los medios, se establecieron los siguientes rangos (más de 15000 , de 5000 a menos de 15000 , de 1000 a menos de 5000 , menos de 1000). Se aplica únicamente a medios impresos.

$\diamond \quad$ Frecuencia: lugar que ocupa la señal del medio en el espectro radioeléctrico. Se aplicó únicamente a medios radiofónicos o televisivos.

$\diamond \quad$ Repetidoras: lugar que ocupa una segunda señal del medio en el espectro. Aplica en casos pertinentes y únicamente para radioemisoras $y$ televisoras.

$\diamond \quad$ Televisión por cable: empresas de cable que transmiten la señal de las empresas televisivas.

$\diamond \quad$ Dirección Web: en caso de contar con una página.

$\diamond \quad$ Dirección de facebook: en caso de utilizar esta red.

$\diamond \quad$ Ubicación del medio: la provincia, el cantón, el distrito y la dirección "a la tica" de cada uno de los medios.
La base de datos que sistematiza y ordena los registros provenientes de fuentes parciales, la revisión Web y de la red social Facebook, constituye un documento básico para la investigación sobre medios de comunicación ${ }^{2}$.

\section{RESULTADOS}

A partir de la definición elaborada para esta investigación, en Costa Rica existen 296 medios de comunicación. Estos se encuentran concentrados principalmente en la provincia de San José con un total de 169 (57\%). Que la provincia en la que se ubica la capital reúna más de la mitad de los medios no es un asunto de importancia menor, pues evidencia que la concentración, en este caso geográfica, es una de las principales características del sistema nacional de medios.

Si bien, en todas las provincias se localizaron medios de comunicación, las diferencias numéricas son significativas: Limón cuenta con 16 medios y Heredia con 13, frente a los 169 que tienen sus oficinas en San José.

2 Las Escuelas de Geografía y de Ciencias de la Comunicación Colectiva de la Universidad de Costa Rica colaboran en un proyecto denominado

\footnotetext{
"Cartografías de la Información”, que permitirá poner a disposición del público, mediante un sitio web, la información en detalle de los medios de comunicación.
} 


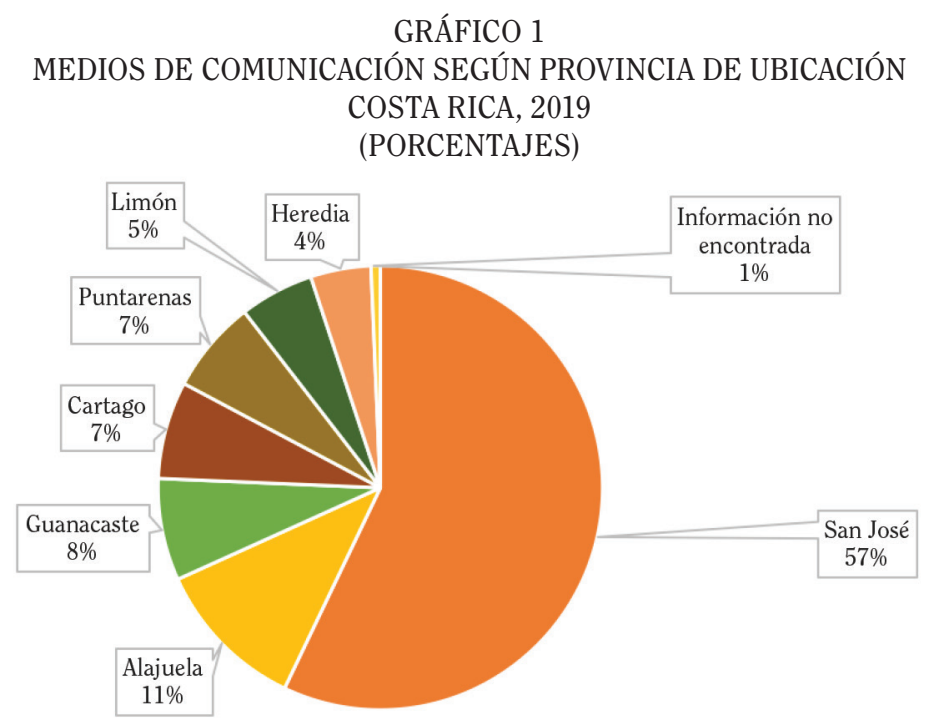

Fuente: $\quad$ Elaboración propia, 2019.

Según se muestra en el gráfico 1, la concentración geográfica de los medios de comunicación se hace aún más evidente cuando se muestra que el cantón central de la provincia de San José reúne a 92 medios de comunicación, esto equivale al $31 \%$ de la totalidad del país.
En la tabla 1, según la plataforma de emisión, se demuestra que dicho cantón emite el $8 \%$ de los canales de cable, el 33\% de los medios digitales, el $41 \%$ de los medios que utilizan el espectro electromagnético y el $19 \%$ de los medios impresos.

TABLA 1

MEDIOS DE COMUNICACIÓN SEGÚN PLATAFORMA DE EMISIÓN Y PORCENTAJE CON RESPECTO AL TOTAL DEL PAÍS CANTÓN DE SAN JOSÉ, 2019

\begin{tabular}{lcc} 
PLATAFORMA DE EMISIÓN & CANTIDAD & DE LOS MEDIOS NACIONALES \\
\hline Cable & 2 & $8 \%$ \\
Digital & 27 & $33 \%$ \\
Espectro radioeléctrico & 50 & $41 \%$ \\
Impresa & 13 & $19 \%$ \\
\hline TOTAL & 92 & $100 \%$ \\
\hline
\end{tabular}

Fuente: $\quad$ Elaboración propia, 2019.

La alta concentración de los medios que utilizan el espectro electromagnético en un territorio tan reducido, permite considerar demandas de actores sociales que han pedido la redistribución del espectro, aprovechando el apagón analógico y el dividendo digital, las cuales son justificadas al considerar que esta concentración impide la incorporación de 
más voces y nuevas agendas en los medios de comunicación.

Por otra parte, la baja concentración relativa (8\%) de canales de televisión que transmiten de forma exclusiva por cable desde San José, es la otra dimensión de la concentración del espectro. Sin acceso a una frecuencia, fuera de la región central han surgido medios de comunicación amparados a las empresas de cable $y$ que se constituyen en iniciativas de carácter local y regional, sobre cuyas condiciones de trabajo y naturaleza se conoce relativamente poco.

En términos de análisis de la concentración geográfica de los medios, cabe destacar que de acuerdo con el estudio realizado, existen hoy día 25 cantones que no cuentan con ningún medio de comunicación. Esto representa casi una tercera parte de los municipios en los que no hay posibilidad de que alguna iniciativa de comunicación esté construyendo una agenda con temas de interés local o que los asuntos públicos de ese cantón puedan ser divulgados $y$ discutidos por sus habitantes. Así, 5 cantones de San José, 7 de Alajuela, 2 de Cartago, 3 de Heredia, 4 de Guanacaste, 3 de Puntarenas y 1 de Limón se encuentran en esa condición, según se presenta en el mapa 1.

MAPA 1

CANTONES QUE NO CUENTAN CON MEDIOS DE COMUNICACIÓN COSTA RICA, 2019

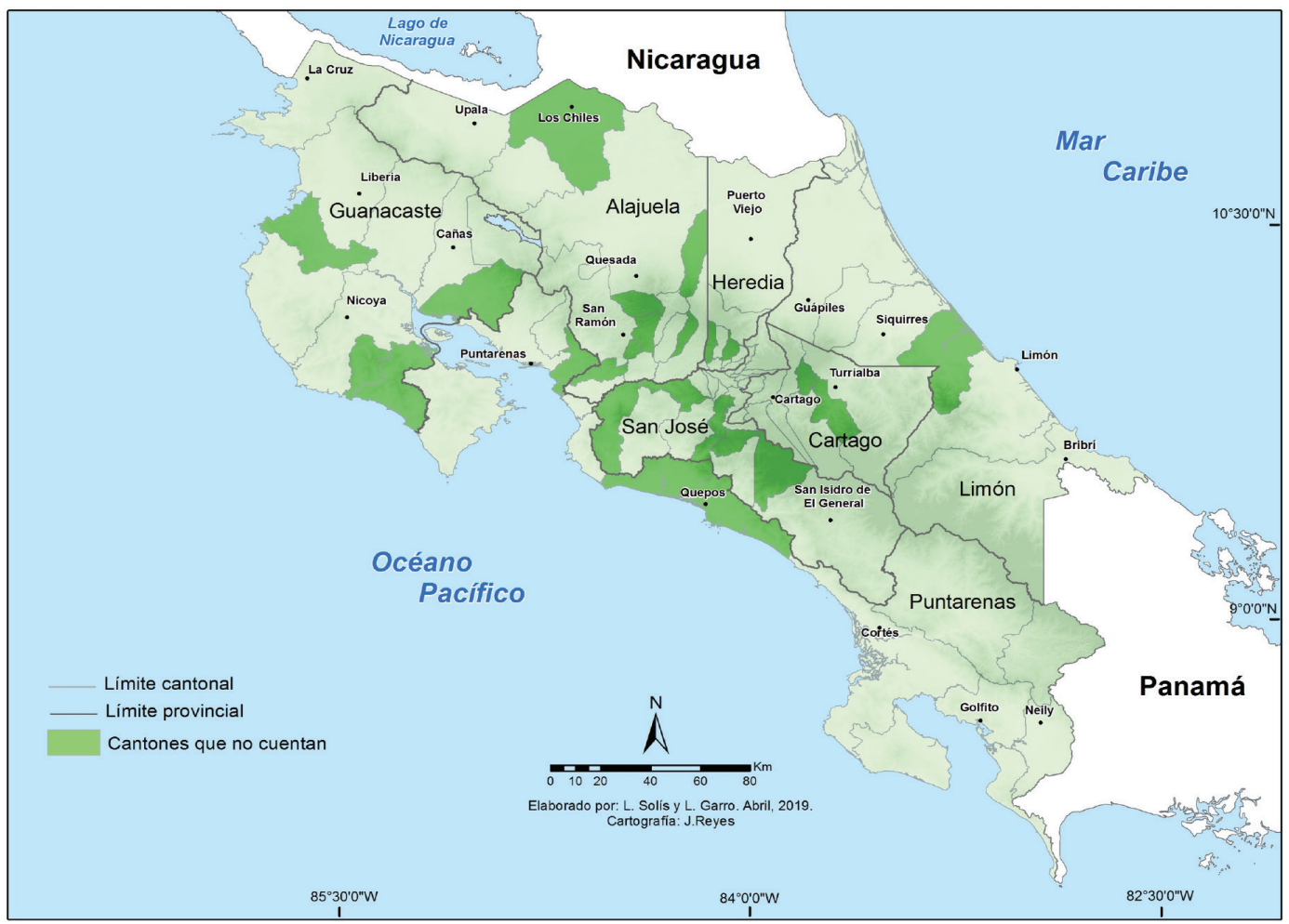

Fuente: Elaboración propia, 2019.

Entre los cantones que cuentan con medios de comunicación, un total de 20 tienen solo uno. Además, hay tipos de medios que son únicos en sus provincias: en
Heredia hay una televisora y en Guanacaste un medio digital; en la provincia de Puntarenas hay un periódico impreso y en Limón una sola revista. 
De esta forma se pone en evidencia una de las características más importantes del sistema mediático costarricense, la alta concentración en la región central del país y la poca presencia mediática más allá del cantón central de San José.

En relación con el tipo de medio de comunicación, sobresale una mayor presencia de medios radiofónicos, con un 43\%, que suman un total de 128 medios. Los medios exclusivamente digitales que nacieron como tales son 23. Los periódicos y las televisoras ajustan números de 57 y 56, respectivamente, con $19 \%$ cada uno de la totalidad de los medios, según se muestra en el gráfico 2 .
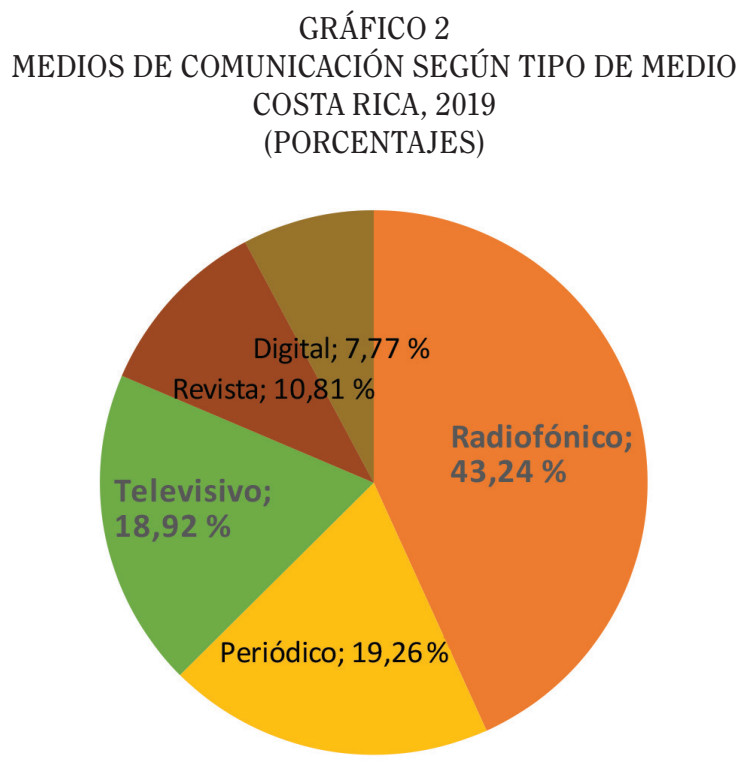

Fuente: $\quad$ Elaboración propia, 2019.

Con respecto a la plataforma de emisión (gráfico 3), 123 medios, equivalentes al 4\%, emiten utilizando el espectro; 81 , correspondientes al 27\%, lo hacen utilizando la plataforma digital; 68 son medios impresos y 24 son canales de televisión por cable. Cabe recordar que, según las definiciones metodológicas establecidas, se clasifican los medios de comunicación según la primera plataforma utilizada. En los casos en que utilizan el espectro radioeléctrico, los medios se clasifican de esa forma, independientemente de que también emitan a través de plataformas digitales. 

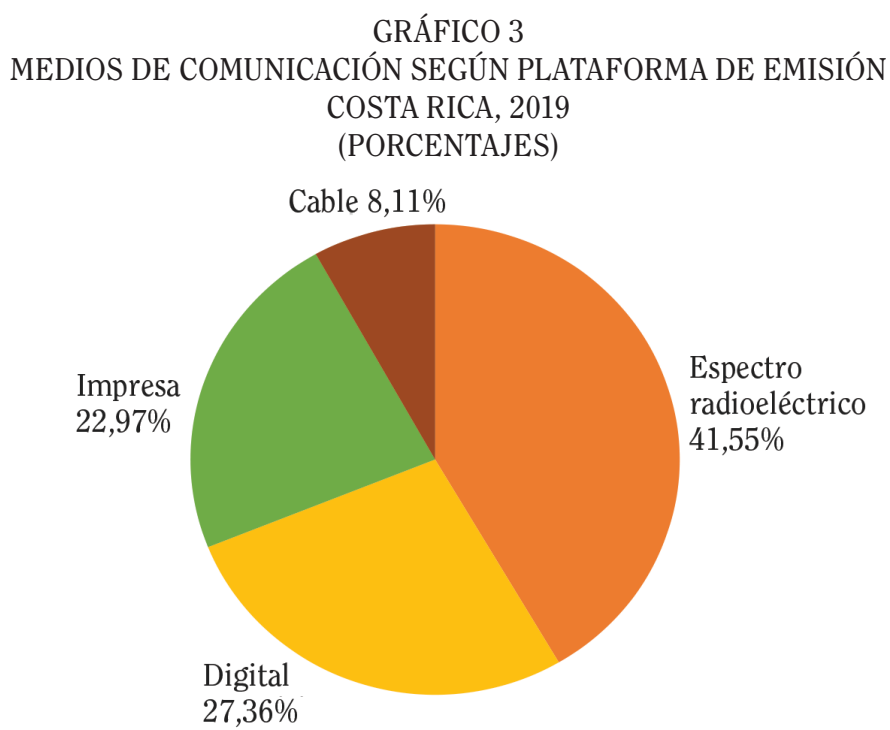

Fuente: Elaboración propia, 2019.

Esta información presenta dos retos de análisis; por una parte, conocer cuál es el uso que los medios de comunicación hacen de las plataformas digitales, entendiendo estas como páginas web y redes sociales; $y$ por otra parte, preguntarse cuáles medios radiofónicos o televisivos que emiten de forma exclusiva sobre las plataformas digitales y el cable están siendo excluidos del uso del espectro radioeléctrico. Ochoa (2018) estableció que una de las características del uso del espectro radioeléctrico en el país es precisamente la concentración y en este sentido, su contraparte sería la exclusión.

En relación con la primera interrogante, destaca que el $97 \%$ del total de los medios de comunicación tienen presencia en las redes sociales a través de Facebook y que el 95\% de los medios cuentan con una página web (ver gráficos 4 y 5).

\section{GRÁFICO 4 \\ MEDIOS DE COMUNICACIÓN SEGÚN TENENCIA DE FACEBOOK \\ COSTA RICA, 2019 \\ (PORCENTAJES)}

Uso de Facebook

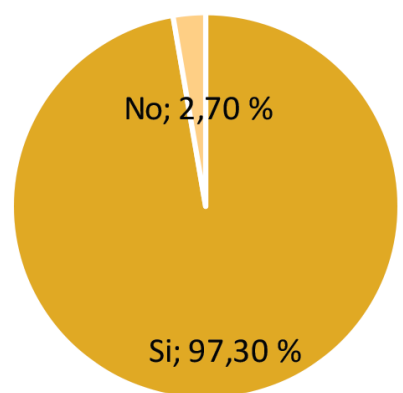

Fuente: $\quad$ Elaboración propia, 2019. 


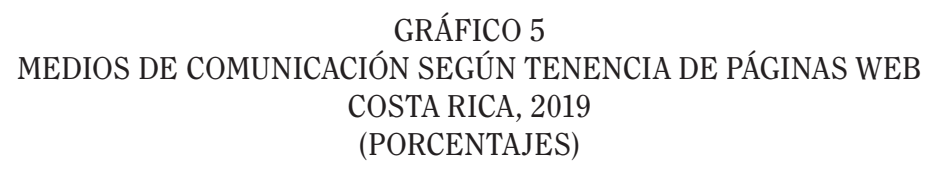

Página Web

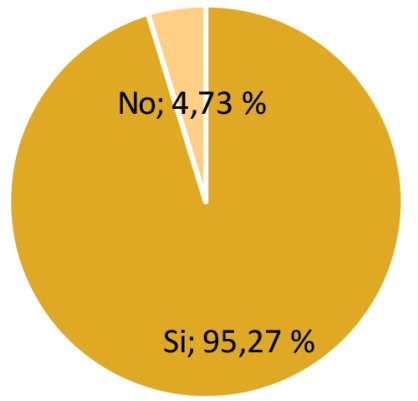

Fuente: $\quad$ Elaboración propia, 2019.

En total, 8 medios de comunicación no tienen una cuenta de Facebook, se trata de 4 periódicos y 4 canales televisivos. Por otra parte, se identificaron 14 medios de comunicación que no cuentan con sitio web propio (4 periódicos, 7 radioemisoras, 1 revista y 2 medios televisivos).

En relación con la segunda interrogante planteada, la tabla 2 facilita reconocer los medios de comunicación que podrían estar siendo excluidos del uso del espectro radioeléctrico.

TABLA 2

TIPO DE MEDIO SEGÚN PLATAFORMA UTILIZADA

COSTA RICA, 2019

\begin{tabular}{|c|c|c|c|c|c|}
\hline & CABLE & DIGITAL & $\begin{array}{c}\text { ESPECTRO } \\
\text { RADIOELÉCTRICO }\end{array}$ & IMPRESA & TOTAL \\
\hline Digital & 0 & 23 & 0 & 0 & 23 \\
\hline Periódico & 0 & 5 & 0 & 52 & 57 \\
\hline Radiofónico & 0 & 29 & 99 & 0 & 128 \\
\hline Revista & 0 & 16 & 0 & 16 & 32 \\
\hline Televisivo & 24 & 8 & 24 & 0 & 56 \\
\hline TOTAL & 24 & 81 & 123 & 68 & 296 \\
\hline
\end{tabular}

Fuente: $\quad$ Elaboración propia, 2019.

El 23\% del total de medios radiofónicos (29 medios) transmiten en digital y podrían hacerlo utilizando el espectro.

En cuanto a los medios televisivos, 8 canales transmiten exclusivamente en digital y 24 por cable; juntos suman 32 , que equivale al $57 \%$ del total de los medios televisivos, los cuales podrían hacerlo utilizando el espectro. No es un dato de menor importancia el puntualizar que más de la mitad de los canales de televisión del país no cuentan con acceso al uso del espectro. 
Esta investigación no tenía como intención específica conocer cuáles medios podrían estar o no interesados en utilizar esta plataforma en caso de tener acceso a frecuencias radioeléctricas debido a la migración a la televisión digital y el dividendo que conlleva un aumento en la cantidad de frecuencias disponibles; sin embargo, se mencionan estos datos con la finalidad de aportar al conocimiento y al análisis más profundo que se requiere como sociedad y para futuras investigaciones.

Enfocada la mirada del análisis en la distribución del uso del espectro radioeléctrico según provincia, se muestra nuevamente la concentración (gráfico 6). En total, 78 de los 123 medios que están haciendo un uso efectivo del espectro, se encuentran en la provincia de San José, lo cual representa el 63\% del espectro utilizado.

\section{GRÁFICO 6 \\ MEDIOS DE COMUNICACIÓN POR PLATAFORMA UTILIZADA SEGÚN PROVINCIA COSTA RICA, 2019}

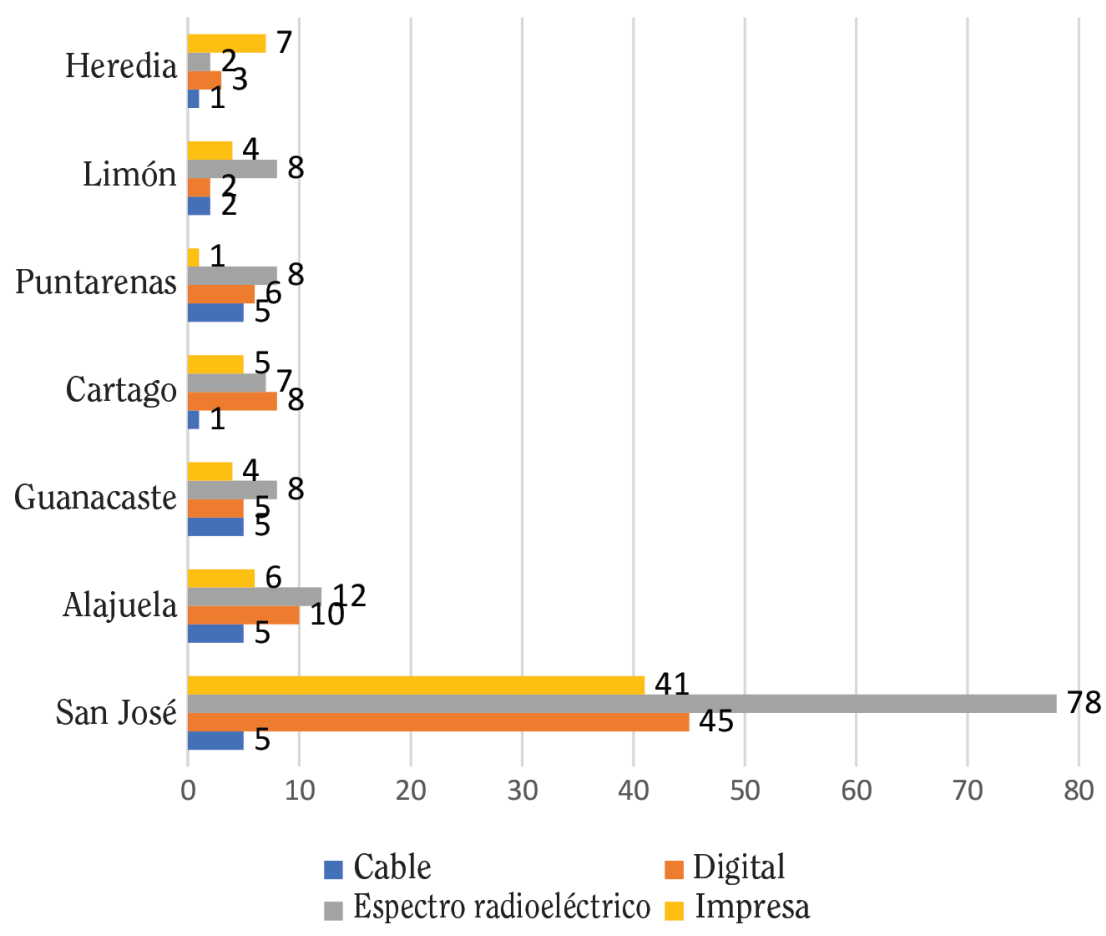

Fuente: $\quad$ Elaboración propia, 2019.

El gráfico 6 muestra la concentración de medios en la provincia de San José en diversas plataformas. Además, en Cartago y Alajuela los medios de comunicación digital son numéricamente muy importantes, si se comparan con aquellos que utilizan el espectro radioeléctrico. Cabe destacar que la distribución de televisoras que funcionan exclusivamente por cable es igual (5 en total) en 4 de las 7 provincias, lo que hace pensar que se requiere democratizar el espectro para que la población que no pueda suscribirse a un servicio pagado, tengan acceso a la televisión digital abierta. 


\section{MEDIOS LOCALES Y MEDIOS NACIONALES}

Como se indicó al comienzo de este artículo, se verificó la existencia de 296 medios de comunicación, de los cuales, 171 (58\%) se reconocen a sí mismos como medios de co- municación nacionales. Ya sea por el alcance, la tecnología de emisión o los contenidos que abordan, un total de 125 (42\%) se consideran a sí mismos medios de carácter local o regional (grafico 7).

\section{GRÁFICO 7 \\ MEDIOS DE COMUNICACIÓN SEGÚN TIPO DE COBERTURA \\ COSTA RICA, 2019 \\ (PORCENTAJES)}

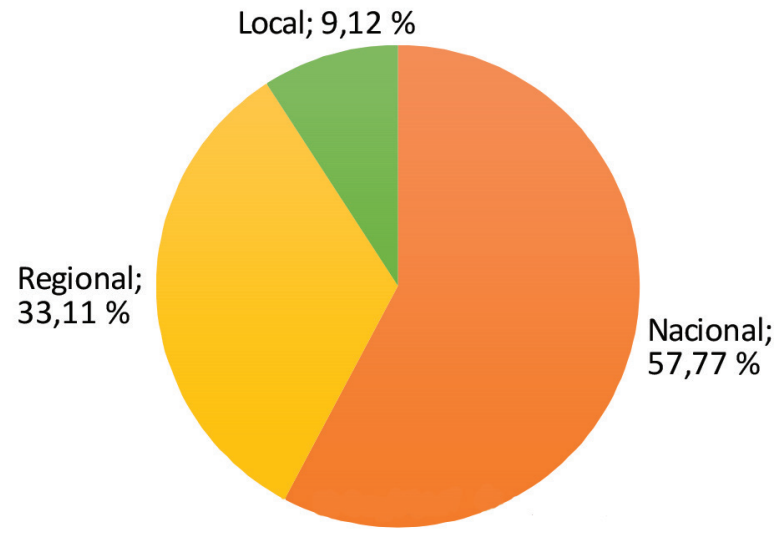

Fuente: Elaboración propia, 2019.

Este dato de 125 medios de comunicación locales o regionales se aleja significativamente de los 71 encontrados por Carazo en el 2014. Las razones de la amplia diferencia no son claras; sin embargo, es sorprendente la coincidencia que presentan ambos estudios en la ubicación de los medios de comunicación por provincia, según se muestra en la tabla 3 .

TABLA 3

MEDIOS REGIONALES Y LOCALES SEGÚN PROVINCIA Y AÑO DE ESTUDIO COSTA RICA, 2014 Y 2019

(PORCENTAJES)

\begin{tabular}{lcc}
\hline PROVINCIA & 2014 & 2019 \\
\hline San José & $24,2 \%$ & $27,2 \%$ \\
Alajuela & $16,9 \%$ & $16,8 \%$ \\
Guanacaste & $19,7 \%$ & $13,6 \%$ \\
Cartago & $8,4 \%$ & $8,8 \%$ \\
Puntarenas & $12,6 \%$ & $13,6 \%$ \\
Limón & $8,5 \%$ & $11,0 \%$ \\
Heredia & $9,7 \%$ & $9,0 \%$ \\
\hline TOTAL & $100 \%$ & $100 \%$ \\
\hline
\end{tabular}

Fuente: Elaboración propia a partir de datos recolectados (2019) y Carazo (2014). 
En otros aspectos, los datos de ambas investigaciones no son comparables debido a que la categorización utilizada es diferente; únicamente es posible comparar el uso de las redes sociales, con las cuales se comprueba que la cantidad de medios que no las utiliza disminuyó en cinco años en 11 puntos porcentuales, pasando del $14 \%$ al $3 \%$.
Como se puede observar en el gráfico 8 , en las regiones y en el nivel local, las iniciativas de comunicación como periódicos y radioemisoras son las que existen en mayor número, con una presencia de 45 y 42 , respectivamente. Contrariamente, solo 3 medios de comunicación digitales se consideran de carácter local o regional.

\section{GRÁFICO 8 \\ MEDIOS DE COMUNICACIÓN LOCALES Y REGIONALES SEGÚN TIPO DE MEDIO \\ COSTA RICA, 2019}

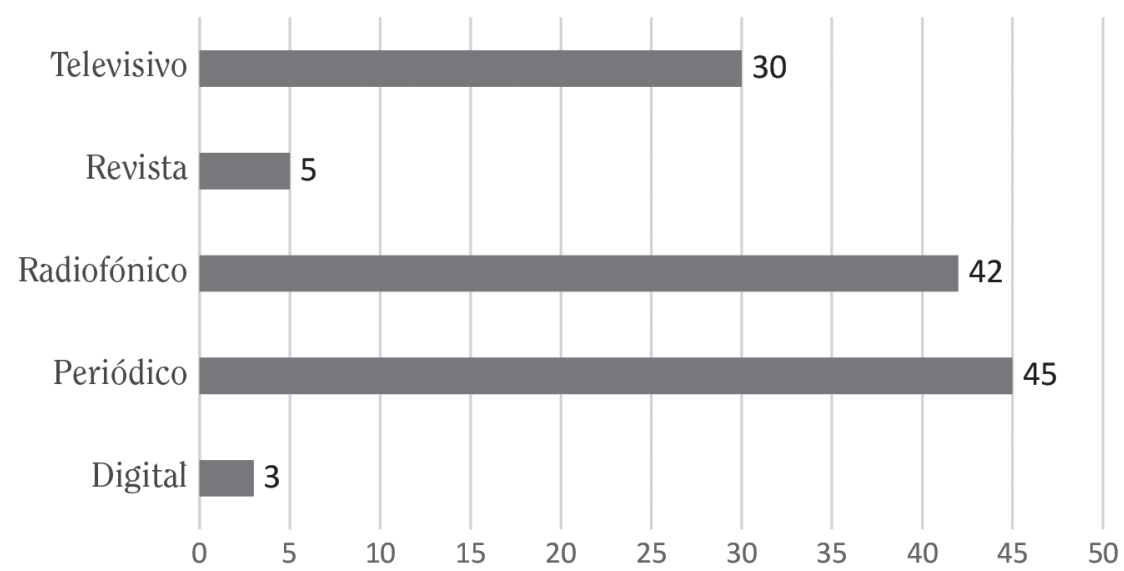

Fuente: $\quad$ Elaboración propia, 2019.

Desde los alcances de esta investigación no son claras las razones por las cuales no existe un mayor número de medios locales o regionales que funcionen de forma exclusiva en la plataforma digital.
Las dificultades de acceso al uso del espectro por parte de las televisoras regionales, podría ser la razón por la que en algunas de estas zonas se utilice mayormente el cable como forma de emisión, tal y como se observa en el gráfico 9 . 

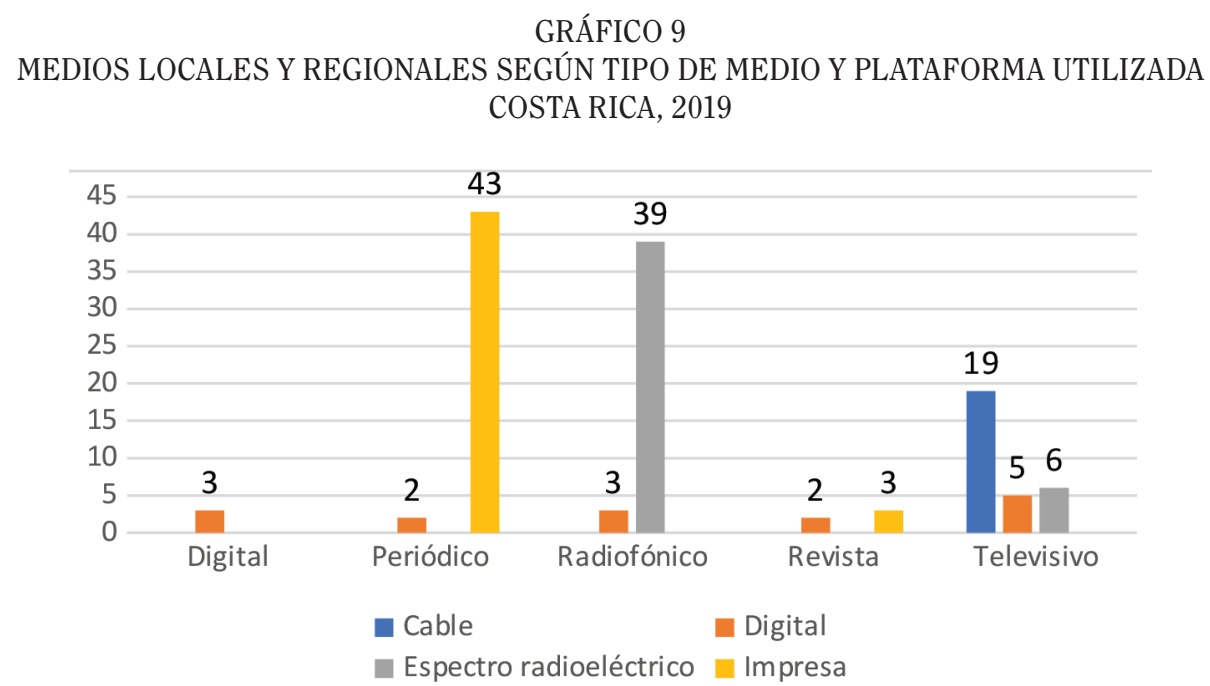

Fuente: $\quad$ Elaboración propia, 2019.

De 30 medios de comunicación televisivos, 19 utilizan como plataforma de emisión las empresas de cable, 5 transmiten en forma digital y únicamente 6 utilizan el espectro. Es decir, de los medios que se autoperciben como locales o regionales, solo el $20 \%$ está transmitiendo su señal utilizando el espectro electromagnético. En este caso, se puede considerar que 24 medios televisivos regionales son usuarios potenciales de nuevas frecuencias de televisión, en caso de que ello fuera posible en el marco del encendido digital. Una relación diferente en el uso del espectro se da en los medios radiofónicos, 39 lo utilizan y únicamente 3 transmiten en la plataforma digital. La fecha de migración a esta tecnología para las radioemisoras que utilizan el espectro aún no está definida.

\section{CONCLUSIONES}

En Costa Rica existe un vibrante escenario de medios de comunicación que es propio de una democracia que requiere garantizar a la ciudadanía la participación en las discusiones propias y diversas que hacen a una sociedad democrática.

Aunque se considera que existe una amplia gama de medios de comunicación, se ha demostrado en estudios anteriores que estos sufren de concentración, especialmente entre los que utilizan el espectro electromagnético para su emisión. Adicionalmente, en este artículo se muestra que la distribución geográfica de los medios genera procesos de concentración y exclusión.

Los medios de comunicación se concentran de manera contundente en la provincia de San José, y especialmente, en el cantón central de San José, mientras que existen 25 cantones que no cuentan con estos medios. Su población ve peligrar la participación directa y cercana en procesos de construcción ciudadana, control político, vivencia de tradiciones y otros papeles que los medios de comunicación están llamados a favorecer. Además, se trata de localidades que tendrán un acceso limitado a las agendas de los medios producidos en la provincia de San José.

Los datos sobre la concentración geográfica profundizan en la necesidad de impulsar acciones para una definición de la política de inclusión de nuevos actores, con el fin de democratizar el espectro radioeléctrico. Esta exclusión empieza en las deficiencias de la propia legislación costarricense, por lo cual, se hace necesario incluir en el marco jurídico la noción de medio comunitario como un tipo de medio 
cuya propiedad y gestión es responsabilidad de comunidades locales o de sentido.

Una de las características de los medios de comunicación que cabe destacar es el uso extendido de la Internet y las redes sociales. Una importante proporción de los medios registrados han visto posibilitada su existencia gracias al desarrollo de las plataformas de emisión que garantiza la tecnología. Sin embargo, también evidencia una situación en la que una cantidad importante de medios están excluidos del uso del espectro: 29 de 128 (el 23\%) de radioemisoras funcionan exclusivamente por Internet y 32 de 56 (41\%) de canales de televisión lo hacen en forma exclusiva por Internet o el cable. Esta exclusión se acrecienta en el caso de los medios locales y regionales.

En el marco de un proceso en curso de transición a la televisión digital, estos son datos que pueden evidenciar un daño potencial al ideal democrático de contar con medios plurales $y$ diversos que funcionen en igualdad de condiciones.

En relación con el uso de las tecnologías, cabe destacar que entre los medios que se autocalifican como locales o regionales, únicamente 3 (de un total de 27) son medios exclusivamente digitales. Como se indicó, no es posible desde esta investigación dar respuesta a la pregunta de por qué están tan poco extendidos los medios digitales en los contextos regionales o comunitarios. Sin embargo, pueden generarse hipótesis de trabajo para investigaciones futuras, que apunten a las características del alcance y costos de Internet, las brechas tecnológicas en el uso, la concentración de profesionales de la comunicación en zonas urbanas o el modelo de negocios de los medios digitales.

Cualesquiera fueran las razones por las que los medios digitales no se han extendido en los espacios locales y regionales en la misma medida que los otros medios, esto demuestra la actualidad $y$ pertinencia de la disputa social por la distribución del espectro radioeléctrico. Asimismo, demuestra la necesidad de investigación en profundidad sobre el uso que se le da a las redes sociales en los medios de alcance nacional y los regionales o locales: actualización, interacción con las audiencias, tipo de contenidos distribuidos y aportes a la construcción de la propuesta comunicativa del medio.

Se evidencian particularidades en las diferentes escalas territoriales en el uso de los medios; así como, en los procesos de concentración y exclusión, lo cual señala el desafío de contar con información actualizada sobre los medios de comunicación. La investigación sobre estos debe abarcar su totalidad y simultáneamente, generar insumos de análisis en las escalas nacional, regional y local de la comunicación.

Es necesario orientar nuevos estudios para identificar las prácticas comunicativas que promuevan el ejercicio de la libertad de expresión, el derecho a la información pública y que alienten la diversidad y el pluralismo al interior del sistema de medios locales y regionales. Los datos muestran la importancia de hacer del sistema mediático costarricense no solo un tema de investigación académico sino también un asunto de interés para la democracia.

Una de las limitaciones de esta investigación es la dificultad para comparar los datos actuales con los obtenidos en las dos investigaciones precedentes que se propusieron generar una base amplia de información sobre los medios de comunicación en el país. La dificultad se presenta desde el momento en que el concepto de medio de comunicación no es compartido en los diferentes estudios. En ese sentido, es necesario un diálogo académico que permita valorar la propuesta metodológica de la que parte esta investigación, de manera que en el futuro se puedan generar bases de datos que permitan analizar tendencias en el desarrollo mediático, considerando que el ejercicio de la ciudadanía comunicativa pasa por la valoración de los medios de comunicación.

\section{REFERENCIAS}

Araya, L., Avendaño, A. y Carazo, E. (2013) La comunicación como derecho: un acercamiento a la legislación costarricense en el contexto de otras legislaciones latinoamericanas. Anuario de Estudios Centroamericanos, 39, 343-371.

Boza, G. (2014). Regulaciones a la Comunicación en Costa Rica. San José, 
Costa Rica: Centro de Investigación en Comunicación. Recuperado de http:// cicom.eccc.ucr.ac.cr/wp-content/ uploads/2014/08/Regulaciones-a-laComunicacio\%CC\%81n-en-Costa-Rica.pdf

Boza, G. (Comp.) (2015). Regulaciones a la comunicación en Costa Rica: normativa vigente con votos relevantes. San José, Costa Rica: Cicom-Proledi. Recuperado de http://cicom.eccc.ucr.ac.cr/wp-content/uploads/2014/08/Regulaciones-a-laComunicacio\%CC\%81n-en-Costa-Rica.pdf Carazo, C. (2014). Estudio de medios comunitarios en Costa Rica. Prospecciones No.2. Centro de Investigación en Comunicación, Universidad de Costa Rica. Recuperado de http://cicom.eccc. ucr.ac.cr/wp-content/uploads/2018/05/ Estudio-de-medios-comunitarios-enC.R.-2014.pdf

Carazo, C. y Halabí, P. (2017). Una radiografía de la industria de la comunicación en Costa Rica. Encuesta a 100 empresas del sector (2016). Revista Reflexiones, 98(1), 39-60.

Chinchilla, L. (2013). El mundo de la radiodifusión en Costa Rica: lo que dice a ley y lo que sucede en la realidad. Costa Rica: Red de Medios e iniciativas de comunicación alternativas.

Córdoba, M.L. (2008). Democracia comunicativa: nuevas formas para la intervención ciudadana. Anagrama, 6 (12), 79-91. Recuperado de http://www.redalyc.org/ articulo.oa?id=491549027005

Fournier, S.; Jiménez, O. y Ochoa, L. (2018). Concentración y transnacionalización de medios en Costa Rica: Caso Albavisión. Primer Informe del Estado de la libertad de Expresión en Costa Rica. Costa Rica: Universidad de Costa Rica.

Garro-Rojas, L., Monge, A. y Solís, L. (2018). Dimensiones para la evaluación del desarrollo mediático en clave de ciudadanía comunicativa. Primer Informe del Estado de la libertad de Expresión en Costa Rica. Costa Rica: Universidad de Costa Rica.

Mata, M.C. (2006). Comunicación y ciudadanía. Problemas teórico-políticos de su articulación. Revista Fronteiras, 8 (1), 5-15. Recuperado de http://revistas. univerciencia.org/index.php/fronteiras/ article/viewArticle/3125

Ochoa, L. (2018). Concentración y transnacionalización de medios en Costa Rica. Primer Informe del Estado de la libertad de Expresión en Costa Rica. Costa Rica: Universidad de Costa Rica.

Robles, F. y Voorend, K. (2011). Los dueños de la palabra en Costa Rica en un contexto de reforma neoliberal. Revista Rupturas, 1(2), 144-161.

Rodríguez, J. (2018). Asignación de la publicidad oficial en los medios de comunicación nacionales. Primer Informe del Estado de la libertad de Expresión en Costa Rica. Costa Rica: Universidad de Costa Rica.

Sandoval, C. y Al-Ghassani, A. (1990). Inventario de los medios de comunicación en Costa Rica. San José (Costa Rica): Escuela de Ciencias de la Comunicación Colectiva, Universidad de Costa Rica.

Lovatón, D. (2014). El equilibrio interamericano entre pluralidad de información y concentración de medios. Derecho PUCP, (73), 131-153. Recuperado de http://www. corteidh.or.cr/tablas/r33401.pdf

Mastrini, G y Becerra, M. (2009). Los monopolios de la verdad en Centroamérica. Buenos Aires, Argentina: Prometeo Libros.
Fecha de ingreso: $28 / 05 / 2019$ Fecha de aprobación: 27/09/2019 
